\title{
Assessing Clinically Meaningful Hypercoagulability after COVID-19 Vaccination: A Longitudinal Study
}

\author{
Elena Campello ${ }^{10}$ Cristiana Bulato ${ }^{1}$ Chiara Simion ${ }^{1}$ Luca Spiezia ${ }^{10}$ Claudia Maria Radu ${ }^{1}$ \\ Sabrina Gavasso ${ }^{1}$ Francesca Sartorello ${ }^{1}$ Graziella Saggiorato ${ }^{1}$ Patrizia Zerbinati ${ }^{1}$ Mariangela Fadin ${ }^{1}$ \\ Daniela Tormene ${ }^{1}$ Paolo Simioni ${ }^{1}$
}

${ }^{1}$ General Internal Medicine and Thrombotic and Hemorrhagic
Diseases Unit, Department of Medicine, Padova University Hospital,
Veneto, Italy

Thromb Haemost 2022;122:1352-1360.
Address for correspondence Paolo Simioni, MD, PhD, General Internal Medicine and Thrombotic and Hemorrhagic Diseases Unit, Department of Medicine (DIMED), Padua University Hospital, Via Giustiniani, 2, Padua 35128, Italy (e-mail: paolo.simioni@unipd.it).

\section{Introduction}

Over the past months, there have been several reports of infrequent thrombotic events associated with coronavirus disease 2019 (COVID-19) vaccines. ${ }^{1-5}$ These thrombotic complications have been well defined within a new syndrome coined vaccine-induced immune thrombotic thrombocytopenia (VITT) characterized by thrombosis, in received

November 20, 2021 accepted after revision January 14, 2022 published online May 27, 2022
A large number of daily requests to exclude possible prothrombotic risk factors for coronavirus disease 2019 (COVID-19) vaccines were received. Our aim was to longitudinally evaluate coagulation profiles in a series of healthy subjects who received COVID19 vaccination and assess hypercoagulability thereafter. Volunteers awaiting a first or second dose of either the ChAdOx1 or BNT162b2 vaccine were enrolled. Venous samples were obtained at baseline (before the vaccine) and longitudinally $3 \pm 2$ days (T1) and $10 \pm 2$ days after the vaccine (T2). Global coagulation monitoring was assessed via platelet count, whole blood thromboelastometry and impedance aggregometry, plasma thrombin generation, and anti-platelet factor 4 (PF4)/heparin immunoglobulin $G$ antibodies. One hundred and twenty-two subjects were enrolled (61 [50\%] ChAdOx1 and 61 BNT162b2). The ChAdOx1 cohort showed a slight but transient increase in thrombin generation (mainly endogenous thrombin potential [ETP] with thrombomodulin and ETP ratio) at T1, which promptly decreased at T2. In addition, the second dose of either vaccine was associated with increased thrombin peak, ETP with thrombomodulin, and ETP ratio. At baseline, 3.2\% of the ChAdOx1 cohort and $1.6 \%$ BNT162b2 cohort were positive for PF4/heparin antibodies with a stable titer through T1 and T2. No relevant differences were detected in platelet count and aggregation, or thromboelastometry parameters. No thrombotic or hemorrhagic events occurred. We can confirm that no clinically meaningful hypercoagulability occurred after either vaccine, albeit keeping in mind that thrombin generation may increase in the first days after the second dose of either vaccine and after the first dose of the ChAdOx1 vaccine.
DOI https://doi.org/ 10.1055/a-1788-5206. ISSN $0340-6245$.

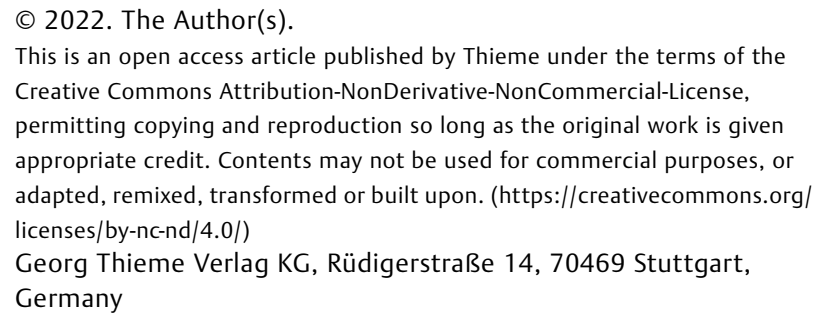
appropriate credit. Contents may not be used for commercial purposes, or adapted, remixed, transformed or built upon. (https://creativecommons.org/ licenses/by-nc-nd/4.0/) Georg Thieme Verlag KG, Rüdigerstraße 14, 70469 Stuttgart, Germany 
particular in the cerebral venous sinus or splanchnic veins. ${ }^{6,7}$ However, this thrombotic complication linked to severe acute respiratory syndrome coronavirus 2 vaccines is extremely rare with an estimated incidence of 0.73 per 100,000 doses of the ChAdOx1 vaccine. ${ }^{8}$ There have been a few reports of vaccine-associated thrombotic events after receiving messenger ribonucleic acid (mRNA) vaccines as well. ${ }^{9-11}$

Although rare, these reports raised profound concern among the general population worldwide. ${ }^{12}$ Our outpatient clinics receive a large number of daily requests from healthy subjects for medical examinations to assess possible risk factors and contraindications for COVID-19 vaccines. Patients with thrombophilic conditions are even more concerned. To date, it remains unclear whether COVID-19 vaccines may induce a transient hypercoagulable state or increase hypercoagulability in subjects with a predisposition to thrombosis with mechanisms other than VITT. A recent observational pilot study by our group found no significant activation of fibrinogen-driven coagulation, plasma thrombin generation (TG), or clinically meaningful platelet aggregation after either ChAdOx1 or BNT162b2. ${ }^{13}$ However, no monitoring of coagulation parameters was done before and after the vaccine.

The aim of the present cohort study was to longitudinally evaluate coagulation profiles in a series of healthy subjects eligible for the BNT162b2 or the ChAdOx1 vaccine and assess hypercoagulability after the vaccination.

\section{Methods}

\section{Study Population and Design}

Volunteers awaiting a first or second dose of either the ChAdOx1 or BNT162b2 vaccine were enrolled. Exclusion criteria were: (1) COVID-19 within the previous 3 months; (2) infection, hospitalization, or surgery within the previous month; (3) pregnancy/postpartum; (4) ongoing anticoagulant therapy; and (5) active cancer (recent diagnosis or radiochemotherapy) in the previous 3 months.

Venous samples were obtained at baseline (T0, at least 2448 hours before the vaccination) and longitudinally $3 \pm 2$ days (T1) and $10 \pm 2$ days after the vaccination (T2). All participants gave written informed consent and the protocol was approved by the national Institutional Ethical Committee INMI Lazzaro Spallanzani (Ref: 366 2020/2021). The study was conducted in compliance with the principles of the Declaration of Helsinki.

\section{Laboratory Tests}

Volunteers underwent fasting venous sampling of $9 \mathrm{~mL}$ of blood into citrate-containing vacutainer tubes. Corn trypsin inhibitor was not used for the samples collection. Plateletpoor plasma (PPP) was prepared within 1 hour by double centrifugation $(2 \times 10$ minute at $1,500 \times g)$ at room temperature. Aliquots $(1 \mathrm{~mL})$ were immediately frozen and then stored at $-80^{\circ} \mathrm{C}$. The following tests were performed:

- platelet count,

- whole blood rotational thromboelastometry (ROTEM),
- whole blood impedance aggregometry,

- TG, and

- anti-platelet factor 4 (PF4)/heparin antibodies.

Platelet count was obtained by an automated cell counter (CELL-DYN Emerald 22, Abbott, Rome, Italy).

ROTEM (ROTEM Instrumentation Laboratory, Werfen, Milan, Italy) was based on the viscoelastic method. Upon activation by calcium, phospholipids, and ellagic acid or tissue factor (TF), clot formation is achieved and thereby decrease the rotational potential of a pin (i.e., viscoelastometry). ${ }^{14}$ The increase in viscoelastic force is proportional to the capability of clot formation in intrinsic (INTEM), extrinsic coagulation (EXTEM) pathways, and fibrinogen contribution to blood clot (FIBTEM). For EXTEM and INTEM tests, the following ROTEM parameters were collected: clotting time (CT), clotting formation time (CFT), and maximum clot firmness (MCF). ${ }^{14,15}$ Only MCF was collected for FIBTEM test.

Platelet aggregation (MULTIPLATE, Roche Diagnostics, Milan, Italy) was based on the impedance method. Upon activation by different agonists, platelets adhere to the sensor wires and thereby increase the electrical resistance (i.e., impedance). The increase is proportional to the capability of platelets to aggregate on each wire. Results are expressed as area under the curve (AUC, AU*min). ${ }^{16}$ The higher the AUC value, the greater the capability of platelets to aggregate. In particular, platelets were stimulated with three different agonists: (1) thrombin receptor activating peptide6 (TRAP-6) $32 \mu \mathrm{mol} / \mathrm{L}$, which is the most potent platelet activator and stimulates platelet aggregation via the thrombin receptor protease-activated receptor-1 (TRAP test, Roche Diagnostics GmbH, Mannheim, Germany); (2) adenosine diphosphate (ADP) $6.5 \mu \mathrm{mol} / \mathrm{L}$ (ADP test, Roche Diagnostics $\mathrm{GmbH}$ ); and (3) arachidonic acid $500 \mu \mathrm{mol} / \mathrm{L}$, which allows the evaluation of cyclooxygenase-dependent aggregation (ASPI test, Roche Diagnostics GmbH). ${ }^{14}$

Thromboelastometry and platelet aggregometry were performed in whole blood within 2 hours of sample collection by trained members of the research team, as previously reported. ${ }^{14,16}$

TG was measured in PPP samples thawed in a $37^{\circ} \mathrm{C}$ water bath for 2 to 3 minutes, using the automated and standardized ST Genesia analyzer (Stago, Asnières sur Seine, France) and the STG-ThromboScreen kit (Stago) according to the manufacturer's instructions. ${ }^{17,18}$ The STG-ThromboScreen kit contains a mixture of phospholipid vesicles ( $4 \mathrm{uM})$ and a medium picomolar concentration (the exact concentration is not specified by the manufacturer) of human recombinant TF with and without rabbit lung thrombomodulin (TM) as activator of the coagulation system. TM concentration is that required to decrease endogenous thrombin potential (ETP) by $50 \%$ in normal pool plasma. The test allows the activation of the protein $C$ anticoagulant pathway, and thus the assessment of both arms of hemostasis. The kit also includes three levels of quality control (for low, normal, and high TM resistance) and a reference plasma to normalize the results and minimize interassay variability. The calibration curve (generated by STG-ThrombiCal) was run in parallel with STG- 
FluoSet allowing a maximal fluorescence level determination and the calculation of a correction factor according to the optical characteristics of each aliquot of plasma assayed. Calibration curve, quality controls, and reference plasma were run on each day of testing. TG was initiated by dispensing a solution of a thrombin fluorogenic substrate and calcium chloride (STG-FluoStart). Analyses with and without TM were performed in duplicate. The following TG parameters were measured and analyzed: lag time, peak thrombin (peak height), and ETP. Lag time and peak height variables were reported as normalized values and expressed as ratio or percentage, respectively. ETP in the presence and absence of TM was provided as absolute value ( $\left.\mathrm{nM}^{*} \mathrm{~min}\right)$. The ETP ratio obtained dividing the ETP with TM by the ETP without TM was also calculated. The ETP ratio reflects the "resistance" to the anticoagulant effect of protein $C$. In other words, the lower the ETP ratio, the better preserved the level and the function of protein C. Conversely, a higher ETP ratio means more severe protein $C$ resistance and a potentially greater predisposition to thrombosis. ${ }^{19}$

The presence of immunoglobulin $G(\operatorname{Ig} G)$ antibodies against PF4/heparin was evaluated with an enzyme-linked immunosorbent assay (ELISA, Asserachrom HPIA-IgG, Stago). All absorbance values greater than the $10.5 \%$ of the absorbance value observed for the reference plasma (containing antibodies that are reactive to heparin-PF4 complexes) were considered positive, as instructed by the manufacturer. The absorbance of the reference plasma was 1.181 optical density (OD), thus normal values were $\leq 0.124$ OD.

\section{Statistical Analysis}

Qualitative data were described as frequencies and percentages. Quantitative data were described as median and interquartile range. Comparisons between dependent groups were performed using the Friedman test for quantitative variables and Fisher's exact tests for frequencies. Comparisons between independent groups were performed using Kruskal-Wallis test. Associations between continuous variables were analyzed using Spearman's correlation tests. Multiple linear regression analysis was run to assess significant association among coagulation parameters and clinical variables (i.e., age, gender, body mass index, vaccine dose [first vs. second], vaccine type, comorbidities, thrombophilia, hormonal therapy, antiplatelet therapy). Statistical significance was set at $p \leq 0.05$. All analyses were completed using SPSS software version 26.0.

\section{Results}

One hundred and twenty-two subjects were enrolled: 61 (50\%) received the ChAdOx1 vaccine and 61 (50\%) the BNT162b2 vaccine. The clinical characteristics of the study population are reported in - Table 1. A comparable number of subjects in each cohort received the first vaccine dose (42 [68\%] ChAdOx1 vs. 36 [59\%] BNT162b2, $p=$ nonsignificant [ns]). The two groups were also comparable as it relates to gender, body mass index, comorbidities, prevalence of known thrombophilia, and ongoing combined oral con- traceptive (COC) therapy. The prevalence of systemic symptoms (i.e., fever, headache, and myalgia/arthralgia/asthenia) was higher in the ChAdOx1 cohort, whereas a higher number of subjects in the BNT162b2 cohort lamented local pain at the injection site. No thrombotic or hemorrhagic eventsincluding petechiae or subcutaneous hematomas-were observed throughout the longitudinal monitoring.

\section{Platelet Count}

We detected a slight but significant decrease in platelet count at $\mathrm{T} 1$ after receiving the ChAdOx1 vaccine followed by a prompt increase at T2 $(198.9 \pm 49.2 \rightarrow 186.1 \pm 48.5 \rightarrow$ $\left.216 \pm 51.3 \times 10^{9} / \mathrm{L} ; p<0.0001\right)$. No significant difference in platelet count was detected after the BNT162b2 vaccine. Furthermore, no significant difference was detected between the two vaccine cohorts (-Table 2 ). In a multiple regression analysis, although platelet count at $\mathrm{T} 1$ was significantly associated with anti-PF4/heparin IgG antibodies ( $p$ 0.032), it was not associated with vaccine type or dose (first vs. second). Importantly, the use of antiplatelet therapy (including nonsteroidal anti-inflammatory drugs [NSAIDs], serotonin reuptake inhibitors, and monoclonal antibodies) was not associated with the decrease of platelet count at T1 after ChAdOx1 vaccine.

\section{Thromboelastometry}

We detected a slight but significant shortening of CFT at T2 after both ChAdOx1 and BNT162b2 vaccines; the shortening was more pronounced in the former cohort (-Table 3 ). No significant difference in other parameters (i.e., CT and MCF) was detected after vaccination and between the two vaccine cohorts.

\section{Whole Blood Platelet Aggregometry}

A significant decrease in ADP- and ASPI-induced platelet aggregation was detected at $\mathrm{T} 1$ after the ChAdOx1 vaccine, then returning to baseline levels at T2 (-Table 4). This phenomenon did not occur after the BNT162b2 vaccine. Moreover, no difference was observed between the two vaccine cohorts at all three time points. ADP- and ASPIinduced aggregation correlated significantly with platelet count ( $r=0.35$ and 0.31 , respectively). In a multiple variable analysis, the use of antiplatelet therapy (including NSAIDs, serotonin reuptake inhibitors, and monoclonal antibodies) was not associated with the decrease of ADP- and ASPIinduced aggregation at $\mathrm{T} 1 \mathrm{after} \mathrm{ChAdOx} 1$ vaccine.

\section{Thrombin Generation}

We detected a significant but transient increase in peak height, ETP with and without TM, and ETP ratio at T1 after the ChAdOx1 vaccine followed by a prompt return to baseline levels at T2 (-Table 5). A similar pattern did not occur after the BNT162b2 vaccine. In a multiple regression analysis, the only parameters independently associated with thrombin peak height were vaccine dose (first vs. second) and COC use at baseline and T1 ( $p=0.025$ and 0.0001 , respectively); only COC use remained significantly associated with peak height $(p=0.0001)$ at T2. There was no association between peak 
Table 1 Clinical characteristics of the study population

\begin{tabular}{|c|c|c|c|}
\hline & ChAdOx1 & BNT162b2 & $p$-Value \\
\hline Number of subjects & 61 & 61 & \\
\hline First dose, $n(\%)$ & $42(68)$ & $36(59)$ & 0.09 \\
\hline Age, y & $51 \pm 12$ & $48 \pm 17$ & ns \\
\hline Gender, female, $n(\%)$ & $47(77.0)$ & $41(67.2)$ & $\mathrm{ns}$ \\
\hline BMI, $\mathrm{kg} / \mathrm{m}^{2}$ & $24.2 \pm 3.6$ & $25.3 \pm 4.1$ & $\mathrm{~ns}$ \\
\hline \multicolumn{4}{|l|}{ Comorbidities (\%) } \\
\hline Hypertension & $16(26.2)$ & $17(27.8)$ & ns \\
\hline Diabetes & $1(1.6)$ & $3(4.9)$ & \\
\hline Dyslipidemia & $8(13.1)$ & $11(18.0)$ & \\
\hline Autoimmune diseases & $5(8.2)^{c}$ & $12(19.6)^{d}$ & \\
\hline Past cancer & $4(6.5)$ & $2(3.3)$ & \\
\hline Known thrombophilia, $n$ (\%) & $7(11.5)^{a}$ & $8(13.1)^{b}$ & $\mathrm{~ns}$ \\
\hline COC therapy, $n(\%)$ & $4(6.5)$ & $4(6.5)$ & ns \\
\hline Antiplatelet therapy, $n$ (\%) & $3(4.9)$ & $3(4.9)$ & ns \\
\hline NSAIDs, (\%) & $4(6.5)$ & 0 & ns \\
\hline \multicolumn{4}{|l|}{ Symptoms, n (\%) } \\
\hline Local & $4(6.5)$ & $13(21.3)$ & 0.02 \\
\hline Fever & $10(16.3)$ & $6(9.8)$ & ns \\
\hline Headache & $11(18.0)$ & $5(8.2)$ & ns \\
\hline Myalgia/arthralgia/asthenia & $14(22.9)$ & $11(18.0)$ & ns \\
\hline Diarrhea & $1(1.6)$ & - & \\
\hline
\end{tabular}

Abbreviations: BMI, body mass index; COC, combined oral contraceptives; MTHFR, methylenetetrahydrofolate reductase; ns, nonsignificant; NSAIDs, nonsteroidal anti-inflammatory drugs.

Note: Data are reported as mean and standard deviation or frequencies.

${ }^{a}$ Two heterozygous prothrombin G20210A mutation, 1 heterozygous factor V Leiden, 1 proteins S deficiency, 2 homozygous MTHFR, 1 antiphospholipid antibody syndrome.

${ }^{\mathrm{b}}$ Six heterozygous factor V Leiden, 1 heterozygous prothrombin G20210A mutation, 1 combined heterozygous (prothrombin G20210A mutation and factor $\mathrm{V}$ Leiden).

'One Raynaud syndrome, 4 Hashimoto's thyroiditis.

'Six Hashimoto's thyroiditis, 1 Sjögren's syndrome, 1 psoriasis, 1 spondyloarthritis, 2 immune thrombocytopenia, 1 Crohn's disease.

Table 2 Platelet count in the study population

\begin{tabular}{|l|l|l|l|l|}
\hline & Platelet count T0 & Platelet count T1 & Platelet count T2 & $p$-Value \\
\hline ChAdOx1 & $198.9 \pm 49.2$ & $186.1 \pm 48.5$ & $216.0 \pm 51.3$ & $<0.0001$ \\
\hline BNT162b2 & $200.0 \pm 54$ & $200.9 \pm 51.2$ & $208.5 \pm 50.8$ & $\mathrm{~ns}$ \\
\hline$p$ between vaccine groups & - & 0.11 & 0.41 & \\
\hline
\end{tabular}

Abbreviation: ns, nonsignificant.

height and type of vaccine. ETP was significantly associated only with COC use $(p=0.001)$ but not with type of vaccine or dose. On the other hand, ETP with TM was significantly and independently associated with vaccine dose and COC use at baseline ( $p=0.004$ and 0.0001 , respectively); with vaccine dose, vaccine type $(p=0.019)$ and COC use at T1; and only with COC use at T2. Similarly, ETP ratio was associated with vaccine dose and COC use at baseline $(p=0.005$ and 0.0001 , respectively); with vaccine dose, vaccine type $(p=0.001)$, and $\mathrm{COC}$ use at $\mathrm{T} 1$; and only with COC use at T2. These results indicate that the type of vaccine increases ETP ratio (by increasing ETP $+\mathrm{TM}$ ) at T1; whereas vaccine dose (first vs. second) increases thrombin peak at baseline and $\mathrm{T} 1$, as well as ETP ratio (by increasing ETP + TM) at baseline and T1. TG parameters remained unchanged 10 days after the vaccination (T2).

\section{Anti-PF4/heparin Antibody}

Overall, anti-PF4/heparin IgG antibodies were detected in $19.6 \%$ (12/61) of the ChAdOx1 cohort and $11.4 \%$ (7/61) of the 


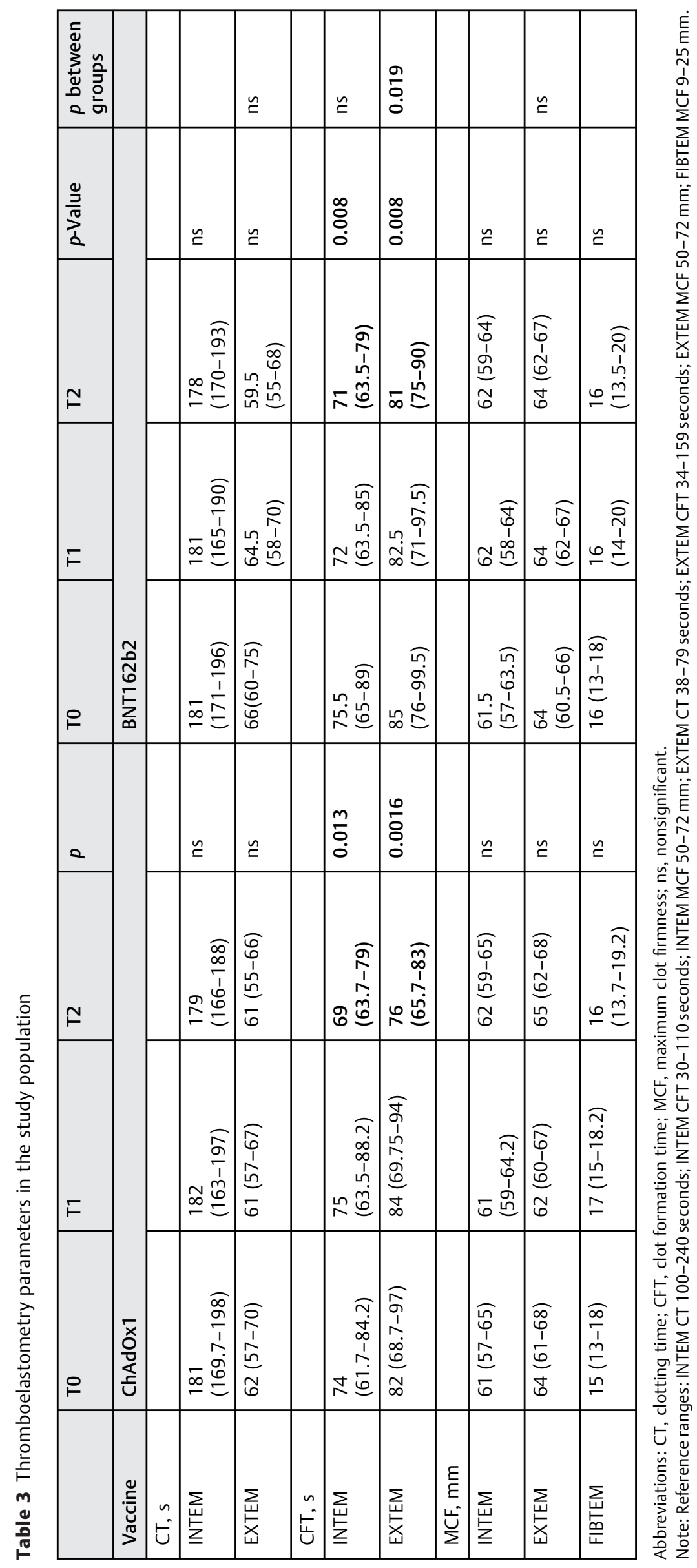

Thrombosis and Haemostasis Vol. 122 No. 8/2022 $\quad$ (c) 2022. The Author(s). 
Hypercoagulability and COVID-19 Vaccine Campello et al. 1357
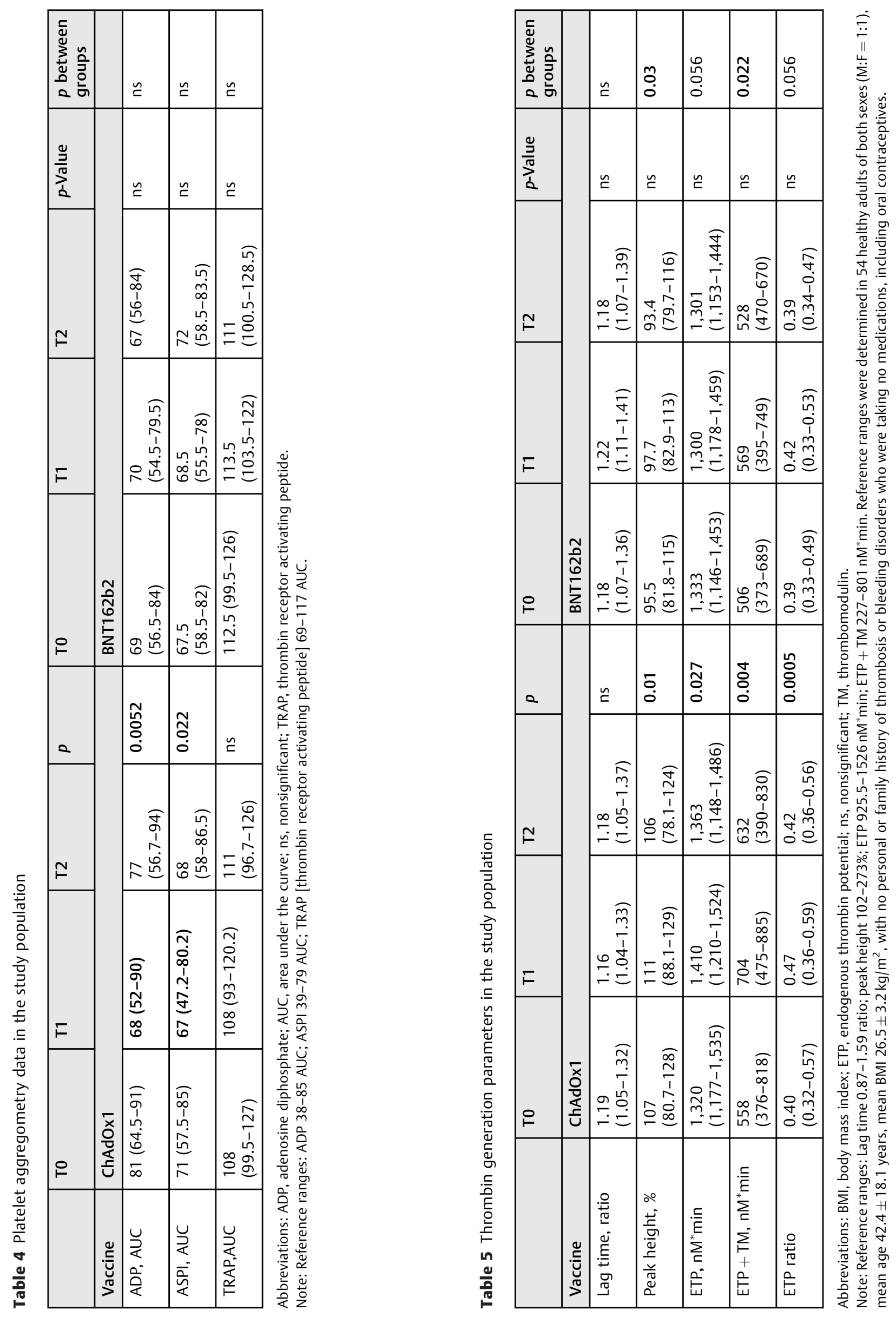

Thrombosis and Haemostasis Vol. 122 No. 8/2022 @ 2022. The Author(s). 


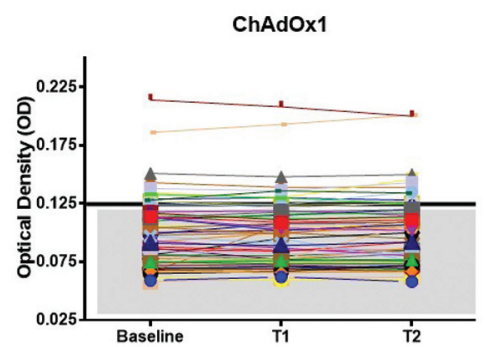

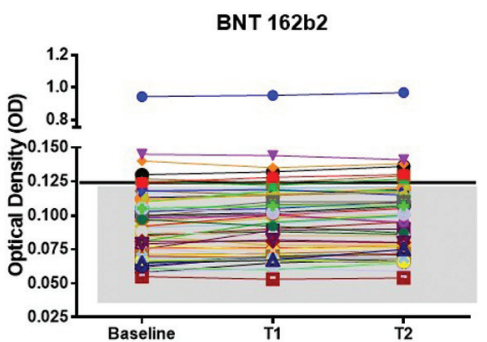

Fig. 1 Trend of platelet factor 4 (PF4)/heparin immunoglobulin G (IgG) antibodies in the study population. (A) Subjects vaccinated with ChAdOx1. (B) Subjects vaccinated with BNT162b2. Values of optical density (OD) $>0.124$ units were considered positive; gray shaded area refers to negative values $(\leq 0.124 \mathrm{OD})$.

BNT162b2 cohort at baseline. However, stronger positivity was present in only $3.2 \%$ (2/61) of the ChAdOx1 cohort and $1.6 \%(1 / 61)$ of the BNT162b2 cohort at baseline, whereas the majority of antibody titers tested weak positive with the method used (- Fig. 1). Particularly, the median levels of anti-PF4 IgG antibodies of positive subjects were 0.136 (0.128-0.151) OD in the ChAdOx1 group and 0.141 (0.1240.344 ) OD in the BNT162b2 group ( $p=n s$ ) (normal values $\leq$ $0.124 \mathrm{OD}$ ). The antibody titer of positive subjects remained stable through T1 (median ChAdOx1 0.136 [0.130-0.148] OD; BNT162b2 0.135 [0.126-0.345] OD; $p=\mathrm{ns)}$ and T2 (median ChAdOx1 0.141 [0.134-0.150] OD; BNT162b2 0.138 [0.128-0.347] OD; $p=n s$ ). Importantly, also the antibody titer of negative subjects remained stable through $\mathrm{T} 1$ and T2. - Fig. 1 reports the trend of anti-PF4/heparin IgG antibodies in the study population with the cutoff for positivity. In the multiple regression analysis, the vaccine dose (first vs. second) was not associated with the presence of antibodies at baseline. Platelet count was associated with detectable antibodies at T1 ( $p$ 0.032), whereas the vaccine type was not associated.

\section{Discussion}

VITT is a rare but severe immunological reaction to the adenoviral vector-based COVID-19 vaccines., ${ }^{6,7}$ Extreme activation of platelets and the coagulation system confer a high risk of death from venous or arterial thrombosis, or secondary hemorrhage. ${ }^{20,21}$ The VITT-associated pathophysiology is rather peculiar and dissimilar to that observed in the development of venous or arterial thrombosis due to more common prothrombotic conditions such as congenital or acquired thrombophilia. ${ }^{20,22}$ The spectre of severe thrombosis as a possible complication of COVID-19 vaccines raised great concern among the general population worldwide, with particular regard to individuals with previous thrombotic complications or thrombophilic conditions. We therefore aimed to allay fears related to thrombotic risk linked to COVID-19 vaccines among clinicians and the population by conducting a longitudinal cohort study using global coagulation assays. A previous pilot study showed that neither adenoviral vector-based nor mRNA-based vaccines resulted in significant changes in thromboelastometry and TG parameters. ${ }^{13}$ We performed the following tests before and after the vaccine: coagulation monitoring, platelet count, and antiPF4/heparin antibody titers.

Our main finding was that the adenoviral vector-based ChAdOx1 vaccine appears to slightly but transiently increase TG mainly in the ETP with TM and ETP ratio parameters 48 hours after the vaccine followed by a sharp decrease at T2. The second dose of either vaccine is associated with increased thrombin peak, ETP with TM, and ETP ratio. No thrombotic events occurred in our population, thus indicating that the transient increase in TG bears no pathological ramifications. This may stem from the transient inflammatory reaction associated with the ChAdOx1 vaccine and, more generally, with the second dose of either vaccine. A higher prevalence of subjects who received the ChAdOx1 vaccine reported fever, myalgia/arthralgia/asthenia, and use of NSAIDs as compared with the BNT162b2 vaccine. This may stem from a more pronounced inflammatory reaction in the former. However, as per the nature of a not blinded observational study, the comparison of such mild side effects may not be so reliable. None of the transient TG alterations were associated with the presence of thrombophilia. In addition, the increased peak height associated with dose (first vs. second) as well as the increase in ETP with TM and ETP ratio associated with dose and vaccine type were independent from COC use. ${ }^{23}$

Our findings on platelet count and platelet aggregation warrant a separate discussion. We detected a slight but significant decrease in platelet count alongside a reduction in platelet aggregation 48 hours after the ChAdOx1 vaccine. The results were confirmed even after removing patients undergoing NSAIDs or any antiplatelet therapy. The slight reduction of platelet function appeared to be associated only with the platelet count. However, both platelet count and aggregation remained within the reference range and did not differ significantly between the two vaccine cohorts.

Thromboelastometry profiles did not reveal any clinically meaningful change after either vaccine. We observed a significant shortening of CFT in INTEM and EXTEM 10 days after both vaccines, though CFT remained within the normal range. No difference in the clot stability (MCF) was detected after either vaccine.

Finally, we detected low titers of anti-PF4/heparin IgG antibodies before the vaccine which remained unchanged after the vaccine. No seroconversion was detected in subjects 
tested negative before the vaccine. The antibody titer was associated with platelet count 48 hours after the vaccine but independently of the vaccine type. The frequency of positive anti-PF4/heparin IgG in our study appears higher than observed in healthy population. ${ }^{24}$ However, we detected a comparable proportion of positive subjects in both the vaccine groups and the antibody titer was low according to the manufacturer cutoff, thus only a similar weak positivity at baseline and no increase in the titer after either vaccination was detected. It has been shown that the specificity of ELISA assays for anti-PF4/heparin IgG antibodies is quite a bit low (74-86\%, depending in part on the assay manufacturer), accounting for a high rate of false positives, also depending on patient population. ${ }^{25,26}$ Our population include also a proportion of subjects with autoimmune diseases, who have a higher likelihood for testing positive because of possible cross-reactivity. ${ }^{25}$ Our results appear to be in contrast with a recent study that detected nonplatelet-activating anti-PF4 antibodies in $67 \%$ of vaccinated individuals on day 22 following the first dose of the ChAdOx1 vaccine, whereas none of the recipients had detectable anti-PF4/heparin IgG antibodies before the vaccine. ${ }^{27}$ Furthermore, anti-PF4 antibodies were also detected after the BNT162b2 vaccine. ${ }^{28}$ In the study by Thiele et al, 7 out of 11 samples tested already positive for anti-PF4/polyanion IgG before vaccination, while four showed "seroconversion" after vaccination with both vaccines. ${ }^{28}$ Importantly, all the aforementioned studies reported only nonplatelet-activating PF4 antibodies in vaccinated individuals. We may therefore conclude that while no PF4/heparin antibodies induced by the vaccines were detected, the latter did not cause an increase in antibody titer even in nonspecific carriers. Thus, there is no clinical indication to screen for PF4/heparin IgG antibodies asymptomatic individuals before and after vaccination.

We would be remiss if we did not mention some of the limitations of our study. Particularly, the number of subjects included is small and this may partly alter the results because of the lack of power. However, in a previous observational pilot study including 190 subjects after the vaccination, we found no difference in the coagulation profile versus a nonvaccinated healthy population. ${ }^{13}$ Moreover, given the small sample size, the multiple regression analysis could be impaired. Finally, we did not perform a functional, plateletactivation assay to clarify the platelet-activating capacity of anti-PF4 antibodies in subjects tested positive or weak positive with the ELISA immunologic assay.

Overall, our findings suggest that: (1) no clinically meaningful hypercoagulability and increase in platelet aggregation occurs after either vaccine; (2) increased TG (peak and ETP ratio) may occur after the second dose of either vaccine (baseline and 48 hours after); (3) transient increase in TG (EPT ratio) occurs 48 hours after the ChAdOx1 vaccine; (4) subjects taking COCs show an independent and stable increase in TG; and (5) high-titer positivity for PF4/heparin antibodies do not occur commonly following vaccination and asymptomatic individuals with (weak) positivity do not increase their titer after vaccination.

These results allow us to fully reassure individuals referred to our center for an assessment of the prothrombotic risk associ- ated with COVID-19 vaccines. In particular, there is no need for coagulation and platelet monitoring after vaccination in individuals asymptomatic for thrombotic or hemorrhagic events.

\section{What is known about this topic?}

- The path mechanism of vaccine-induced immune thrombotic thrombocytopenia has been defined.

- Thrombotic complications associated to COVID-19 vaccination are rare but raised concern in the general population.

- There is no indication to assess hypercoagulability before COVID-19 vaccination.

\section{What does this paper add?}

- No increase thromboelastometry and platelet aggregation occurs after ChAdOx1 or BNT162b2 vaccine.

- Increased thrombin generation may occur after the second dose of either vaccine.

- People taking oral contraceptives or with autoimmune diseases can be reassured on the lack of vaccine-driven hypercoagulability.

\section{Funding}

The study was funded by Ministry of Education, University and research (MIUR), grant DOR2147942/2021 to P.S.

Conflict of interest

None declared.

Acknowledgment

We would like to thank all the volunteer participants who willingly agreed to undergo serial blood draws for research purposes.

\section{References}

1 Greinacher A, Thiele T, Warkentin TE, Weisser K, Kyrle PA, Eichinger S. Thrombotic thrombocytopenia after ChAdOx1 nCov-19 vaccination. N Engl J Med 2021;384(22):2092-2101

2 Schultz NH, Sørvoll IH, Michelsen AE, et al. Thrombosis and thrombocytopenia after ChAdOx1 nCoV-19 vaccination. N Engl J Med 2021;384(22):2124-2130

3 Scully M, Singh D, Lown R, et al. Pathologic antibodies to platelet factor 4 after ChAdOx1 nCoV-19 vaccination. N Engl J Med 2021; 384(23):2202-2211

4 See I, Su JR, Lale A, et al. US case reports of cerebral venous sinus thrombosis with thrombocytopenia after Ad26.COV2.S vaccination, march 2 to April 21, 2021. JAMA 2021;325(24):2448-2456

5 Ceschia N, Scheggi V, Gori AM, et al. Diffuse prothrombotic syndrome after ChAdOx1 nCoV-19 vaccine administration: a case report. J Med Case Reports 2021;15(01):496

6 Klok FA, Pai M, Huisman MV, Makris M. Vaccine-induced immune thrombotic thrombocytopenia. Lancet Haematol 2022;9(01): e73-e80

7 Pavord S, Scully M, Hunt BJ, et al. Clinical features of vaccineinduced immune thrombocytopenia and thrombosis. N Engl J Med 2021;385(18):1680-1689 
8 Chan BTB, Bobos P, Odutayo A, Pai M. Meta-Analysis of Risk of Vaccine- Induced Immune Thrombotic Thrombocytopenia Following ChAdOx1-S Recombinant Vaccine. Accessed March 21, 2022 at: https://www.medrxiv.org/content/10.1101/2021.05. $04.21256613 \mathrm{v} 1$

9 Baden LR, El Sahly HM, Essink B, et al; COVE Study Group. Efficacy and safety of the mRNA-1273 SARS-CoV-2 vaccine. N Engl J Med 2021;384(05):403-416

10 Uaprasert N, Panrong K, Rojnuckarin P, Chiasakul T. Thromboembolic and hemorrhagic risks after vaccination against SARS-CoV2: a systematic review and meta-analysis of randomized controlled trials. Thromb J 2021;19(01):86

11 Sangli S, Virani A, Cheronis N, et al. Thrombosis with thrombocytopenia after the messenger RNA-1273 vaccine. Ann Intern Med 2021;174(10):1480-1482

12 Razai MS, Chaudhry UAR, Doerholt K, Bauld L, Majeed A. Covid-19 vaccination hesitancy. BMJ 2021;373(1138):n1138

13 Campello E, Simion C, Bulato C, et al. Absence of hypercoagulability after nCoV-19 vaccination: an observational pilot study. Thromb Res 2021;205:24-28

14 Spiezia L, Radu C, Campello E, et al. Whole blood rotation thromboelastometry (ROTEM $\left({ }^{\circledR}\right)$ ) in nine severe factor $V$ deficient patients and evaluation of the role of intraplatelets factor $\mathrm{V}$. Haemophilia 2012;18(03):463-468

15 Hartmann J, Walsh M, Grisoli A, et al. Diagnosis and treatment of trauma-induced coagulopathy by viscoelastography. Semin Thromb Hemost 2020;46(02):134-146

16 Zanetto A, Senzolo M, Campello E, et al. Influence of hepatocellular carcinoma on platelet aggregation in cirrhosis. Cancers (Basel) 2021;13(05):1150

17 Campello E, Bulato C, Spiezia L, et al. Thrombin generation in patients with COVID-19 with and without thromboprophylaxis. Clin Chem Lab Med 2021;59(07):1323-1330

18 Morrow GB, Beavis J, Harper S, et al. Coagulation status of critically ill patients with and without liver disease assessed using a novel thrombin generation analyzer. J Thromb Haemost 2020;18(07):1576-1585

19 Zanetto A, Rinder HM, Campello E, et al. Acute kidney injury in decompensated cirrhosis is associated with both hypo-coagulable and hyper-coagulable features. Hepatology 2020;72(04):1327-1340

20 Greinacher A, Selleng K, Palankar R, et al. Insights in ChAdOx1 nCov-19 vaccine-induced immune thrombotic thrombocytopenia (VITT). Blood 2021;138(22):2256-2268

21 McGonagle D, De Marco G, Bridgewood C. Mechanisms of immunothrombosis in vaccine-induced thrombotic thrombocytopenia (VITT) compared to natural SARS-CoV-2 infection. J Autoimmun 2021;121:102662

22 Campello E, Spiezia L, Adamo A, Simioni P. Thrombophilia, risk factors and prevention. Expert Rev Hematol 2019;12(03):147-158

23 Tchaikovski SN, van Vliet HA, Thomassen MC, et al. Effect of oral contraceptives on thrombin generation measured via calibrated automated thrombography. Thromb Haemost 2007;98(06): 1350-1356

24 Greinacher A, Holtfreter B, Krauel K, et al. Association of natural anti-platelet factor 4/heparin antibodies with periodontal disease. Blood 2011;118(05):1395-1401

25 Juhl D, Eichler P, Lubenow N, Strobel U, Wessel A, Greinacher A. Incidence and clinical significance of anti-PF4/heparin antibodies of the IgG, IgM, and IgA class in 755 consecutive patient samples referred for diagnostic testing for heparin-induced thrombocytopenia. Eur J Haematol 2006;76(05):420-426

26 Otis SA, Zehnder JL. Heparin-induced thrombocytopenia: current status and diagnostic challenges. Am J Hematol 2010;85(09):700-706

27 Terpos E, Politou M, Ntanasis-Stathopoulos I, et al. High prevalence of anti-PF4 antibodies following ChAdOx1 nCov-19 (AZD1222) vaccination even in the absence of thrombotic events. Vaccines (Basel) 2021;9(07):712

28 Thiele T, Ulm L, Holtfreter S, et al. Frequency of positive anti-PF4/ polyanion antibody tests after COVID-19 vaccination with ChAdOx1 nCoV-19 and BNT162b2. Blood 2021;138(04):299-303 